Research Paper

\title{
Comparison of treatment plan quality of VMAT for esophageal carcinoma with: flattening filter beam versus flattening filter free beam
}

\author{
Wen-zhao Sun*, Li Chen*, Xin Yang, Bin Wang, Xiao-wu Deng, Xiao-yan Huang ${ }^{\bowtie}$ \\ State Key Laboratory of Oncology in South China, Collaborative Innovation Center for Cancer Medicine, Department of Radiation Oncology, Sun Yat-sen \\ University Cancer Center, Guangzhou, People's Republic of China \\ * These authors contributed equally to this study. \\ $\triangle$ Corresponding author: Xiao-yan Huang, Address at: State Key Laboratory of Oncology in South China, Collaborative Innovation Center for Cancer Medicine, \\ Department of Radiation Oncology, Sun Yat-sen University Cancer Center, 651 Dongfeng Road East, Guangzhou 510060, People's Republic of China. E-mail \\ address: huangxy@sysucc.org.cn (XY.Huang) \\ (C) Ivyspring International Publisher. This is an open access article distributed under the terms of the Creative Commons Attribution (CC BY-NC) license \\ (https://creativecommons.org/licenses/by-nc/4.0/). See http://ivyspring.com/terms for full terms and conditions.
}

Received: 2018.03.13; Accepted: 2018.08.02; Published: 2018.09.07

\begin{abstract}
Purpose: To investigate the difference in treatment plan quality of volumetric modulated arc treatment (VMAT) for esophageal carcinoma with flattening filter beam (FF) and flattening filter free beam (FFF).

Material and methods: A total of fifty-six treatment plans were generated for twenty eight esophageal carcinoma patients with flattening filter beam and flattening filter free beam, using same optimal parameters. The homogeneity index $(\mathrm{HI})$ and conformal index $(\mathrm{Cl})$ of targets, and some special points on Dose-Volume Histogram (DVH) curves were used to compare the plan quality. The coverage volumes of $45 \mathrm{~Gy}, 30 \mathrm{~Gy}$ and $20 \mathrm{~Gy}$ outside targets $\left(\mathrm{V}_{45 \mathrm{G} y}, \mathrm{~V}_{30 \mathrm{~Gy}}\right.$ and $\left.\mathrm{V}_{20 \mathrm{G}}\right)$ were used to compare the targets peripheral dose. The MU numbers, measured delivery time and averaged dose rates were used to evaluate the delivery efficiency of treatment plans.

Results: A significant decreasing in peripheral dose around targets was found using FFF beams while the dose distributions in targets were equivalent to the plans with FF beams. $V_{45 G y}, V_{30 G y}$ and $V_{20 G y}$ were decreased by $6.46 \%, 88.18 \%$ and $4.40 \%$, respectively. A significant increase in MUs and decrease in treatment time were also found in delivery test. The average MUs was increased by $21.83 \%$ and the average treatment time was reduced by down to $11.9 \%$.

Conclusions: For esophageal carcinoma, the research showed that the treatment plans with FFF beams could get comparable dose distribution in targets and could significantly reduce the peripheral dose around targets compared to the plans with FF beams.
\end{abstract}

Key words: flattening filter free beam, esophageal carcinoma, peripheral dose, plan quality

\section{Introduction}

Esophageal carcinoma is the ninth most prevalent cancer in the word [1] and has an estimated 5 -years survival rate of $10 \%$ [2]. Radiotherapy is an import component in the management of esophageal carcinoma for pre-operative and definitive treatment. For esophageal carcinoma, due to complex of planning target volume are surrounded by many organs at risks (OARs) such as spinal cord, lung, heart and liver, radiotherapy has shift from 3D conformal radiation therapy (3D-CRT) to advanced radiotherapy such as intensity modulated radiation therapy (IMRT) and volumetric modulated arc treatment (VMAT) for the clinical benefits of increasing dose to tumor and sparing organs at risk (OARs). Several studies have demonstrated that volumetric modulated arc therapy has the ability to reduce monitor units and treatment time when compared with intensity-modulated radiation therapy [3.4.5.6].

Recently, there has been a growing interesting in operating medical linear accelerators without a 
flattening filter (FFF). The main advantages of FFF beams are increased dose rate, reduced scatter radiation, reduced leaf transmission and treatment head leakage [7]. Numerous literatures reported that the increased dose rate of FFF beams is particularly beneficial for stereotactic body radiotherapy (SBRT) due to reduction of the treatment times of large fraction doses $[8.9,10]$. Plenty of literatures reported that VAMT plan was able to provide similar target coverage for esophageal carcinoma while reducing monitors units and treatment times compared to IMRT plan [11,12]. Gabriele Kragl pointed out that removing the flattening filter lead to reduced peripheral doses for advanced treatment techniques [9]. So it's necessary to study whether benefits exist in esophageal carcinoma radiotherapy using flattening filter free (FFF) compared to flattened (FF) beams. The purpose of this study is to make a well comparison of dosimetric characteristics and delivery efficiency of treatment plan for esophageal carcinoma using flattening filter free (FFF) and flattened (FF) beams.

\section{Materials and Methods}

\section{Patients}

The data of twenty eight esophageal carcinoma patients who underwent VMAT technique under VersaHD unit (Elekta AB, Stockholm, Sweden) were randomized selected from our database and fully anonymized for the purpose of this study. The biopsy results showed that all patients were squamous cell carcinoma. Each patient had received 60Gy for PTV1 and 50Gy for PTV2 in total 28 fractions, with a dose of 2.14Gy given for PTV1 each weekday. The clinical information for each patient is listed in Table 1.

\section{Computed tomography (CT) simulation}

For each patient, the serious CT scans were performed using a Philips Brilliance CT Big Bore (Phillips Medical System, 96 Highland Heights, OH, USA). Patients were immobilized using a body vacuum pillow and scanned with head first and supine on position. The reconstruction slice thickness is $5 \mathrm{~mm}$ and region scanned extended from the cricothyroid membrane to the lower edge of liver. All patients CT images were transmitted to Monaco treatment planning system (TPS) (Monaco V5.0.2, Elekta AB, Stockholm, Sweden) to delineate the target volumes and organs at risk (OARs) and design the treatment plan.

\section{Delineation of anatomy structures}

All organ structures were delineated by trained physician. The gross tumor volume (GTV) of esophageal tumor was including the esophageal tumor and the metastasis lymph nodes. The clinical target volume (CTV) was defined as $3.0 \mathrm{~cm}$ cranial-caudal margin and $1.0 \mathrm{~cm}$ margin in other direction. Regional lymphatic drainages were covered by the CTV, which included the para-esophagus, medial,and upper mediastinal lymph nodes. When the primary tumor was located in the cervical or the supper thoracic esophagus, the supraclavicular lymph nodes were included. For the patients with lower thoracic esophageal carcinoma, the pericardial lymph node was included. Planning target volumes (PTVs), which included PTV1 and PTV2, were generated by $5 \mathrm{~mm}$ outer margin of GTV and CTV. The OARs, including the spinal cord, lung, heart, liver, were contoured following anatomic definitions.

Table 1. Patient's characteristics and target volume.

\begin{tabular}{|c|c|c|c|c|c|}
\hline Patients & Staging & Gender & Age & $\begin{array}{l}\text { PTV1 } \\
\text { volume }\left(\mathrm{cm}^{3}\right)\end{array}$ & $\begin{array}{l}\text { PTV2 } \\
\text { volumes }\left(\mathrm{cm}^{3}\right)\end{array}$ \\
\hline 1 & T2N1M0 & $\mathrm{M}$ & 79 & 125.24 & 410.48 \\
\hline 2 & $\mathrm{~T} 2 \mathrm{~N} 1 \mathrm{M} 0$ & M & 51 & 179.855 & 776.235 \\
\hline 3 & T2N3M0 & M & 53 & 126.425 & 736.19 \\
\hline 4 & $\mathrm{~T} 2 \mathrm{~N} 1 \mathrm{M} 0$ & M & 68 & 117.39 & 447.895 \\
\hline 5 & T2N1M1 & M & 73 & 153.81 & 485.195 \\
\hline 6 & T3N1M1 & M & 51 & 136.54 & 452.54 \\
\hline 7 & T2N1M1 & M & 65 & 183.215 & 602.45 \\
\hline 8 & T3N0M0 & M & 56 & 445.98 & 903.175 \\
\hline 9 & $\mathrm{~T} 2 \mathrm{~N} 3 \mathrm{M} 0$ & M & 61 & 318.14 & 645.775 \\
\hline 10 & T3N1M1 & M & 58 & 282.46 & 823.08 \\
\hline 11 & T3NIM0 & M & 49 & 184.98 & 611.405 \\
\hline 12 & T2N1M1 & M & 51 & 502.045 & 961.765 \\
\hline 13 & T2N1M0 & M & 64 & 91.475 & 572.33 \\
\hline 14 & T2N3M0 & M & 64 & 149.155 & 754.925 \\
\hline 15 & T2N1M0 & M & 61 & 286.28 & 804.935 \\
\hline 16 & T4N0M0 & M & 59 & 225.27 & 661.485 \\
\hline 17 & T3N1M1 & M & 45 & 82.031 & 644.185 \\
\hline 18 & T4N1M0 & M & 62 & 125.895 & 570.99 \\
\hline 19 & T3N1M0 & $\mathrm{F}$ & 49 & 135.765 & 616.805 \\
\hline 20 & T2N1M0 & M & 75 & 54.685 & 443.09 \\
\hline 21 & T3N2M0 & M & 78 & 52.975 & 192.575 \\
\hline 22 & T3N1M1 & M & 63 & 101.3 & 642.26 \\
\hline 23 & T4N1M0 & M & 68 & 200.68 & 701.775 \\
\hline 24 & T2N1M0 & M & 67 & 418.695 & 975.74 \\
\hline 25 & T3N3M0 & M & 57 & 91.605 & 449.025 \\
\hline 26 & T4N1M0 & M & 79 & 145.1 & 526.84 \\
\hline 27 & T3N1M1 & M & 66 & 84.085 & 472.97 \\
\hline 28 & T3N1M1 & M & 60 & 62.34 & 509.59 \\
\hline Mean & & & 61.8 & 184.4 & 621.3 \\
\hline
\end{tabular}

\section{Radiotherapy plans}

A VMAT plan with FFF beam was created by directly changing the original plan radiation energy 6MV (6 Megavoltage with FF beam) to 6FFF (6 MV with FFF beam) and maintaining the original plan optimization parameters unchanged. And then the optimization was performed inversely using original plan parameters and dose were calculated using Monte Carlo (MC) algorithm with 3\% of dose statistical uncertainty per control point and $3 \mathrm{~mm}$ of voxel grid size. All original VMAT plans used a single full arc of $360^{\circ}$ gantry rotation from -180 to 180 in clockwise direction. The max dose rate was set to 600 $\mathrm{MU} / \mathrm{min}$ for $6 \mathrm{MV}$ and $1400 \mathrm{MU} / \mathrm{min}$ for $6 \mathrm{FFF}$. The 
couch angle was set to 0 degree. And collimator was set to $15^{\circ}$ to minimize the contribution of tongue and groove effect during the arc rotation.

\section{Plan evaluation}

All plans were produced with Monaco treatment planning system (Monaco V5.0.2, Elekta AB, Stockholm, Sweden) and this allowed for all dose volume histograms (DVHs) to be obtained with the same sampling algorithm and evaluation of plans based on the standard DVH. For the PTVs, the parameters analyzed were D $2 \%, \mathrm{D} 98 \%, \mathrm{~V}{ }_{100 \%}, \mathrm{~V} 95 \%$, and homogeneity index (HI) and conformal index (CI). The algorithm for CI (19) and HI (18) were described as following.

$$
C I_{95 \%}=\frac{P T V_{95 \%}}{P T V} \times \frac{P T V_{95 \%}}{V_{95 \%}}
$$

$\mathrm{PTV}_{95 \%}$ is the planning target volume that received $95 \%$ of the prescribed dose, PTV is the planning target volume and $\mathrm{V}_{95 \%}$ is the volume that received $95 \%$ of the prescribed dose.

$$
H I=\frac{D_{5 \%}}{D_{95 \%}}
$$

$\mathrm{D}_{5 \%}$ and $\mathrm{D}_{98 \%}$ indicate the doses that covered 5\% and $98 \%$ of the PTV volume, respectively.

For the OARs, the parameters analyzed included as following: (1) Lung: the volume of Lung receiving dose at least 30, 20 and 10 Gy $\left(\mathrm{V}_{30 \mathrm{~Gy}}, \mathrm{~V}_{20 \mathrm{~Gy}}, \mathrm{~V}_{10 \mathrm{~Gy}}\right)$;(2)Heart: the volume of heart receiving dose at least 40 and $30 \mathrm{~Gy}\left(\mathrm{~V}_{40 \mathrm{~Gy}}, \mathrm{~V}_{30 \mathrm{~Gy}}\right)$, and mean heart dose $\left(\mathrm{D}_{\text {mean }}\right)$;(3)Spinal cord: the maximum dose covering $1 \mathrm{cc}$ volume of the spinal cord $\left(\mathrm{D}_{1 \mathrm{cc}}\right.$.

Peripheral doses around PTVs were analyzed via comparison of volume outside PTV2 covered by 45 Gy, 30 Gy, and 20 Gy $\left(\mathrm{V}_{45 G y}, V_{30 G y}, V_{20 G y}\right)$.

\section{Delivery efficiency comparison}

Machine units (MUs) and treatment times were collected for delivery efficiency comparison, and median dose rates were also abstracted from log file for comparison. For VersaHD unit, the operation system (Version 3.1) contain one sub function named record graphic, which can record the information about actual delivery for chosen key words with the sample frequency of 4 times per second and can saved data as .xls format.

\section{Statistical analysis}

The SPSS 19.0 software (IBM, Chicago, IL) was used for statistical data management and analysis. To determine statistical significance, the paired-sample $T$ test was performed with $P$ values $<0.05$ considered significant. Student $\mathrm{t}$ test was also performed. Data are presented as the mean over all patients with standard deviations (SD).

\section{Results}

\section{Patient characteristics}

Twenty eight esophageal carcinoma patients ( 27 men, 1 female) were selected. The median age was 61.8 years (range, 45-79years). According to the sixth edition of the classification by the International Union Against Cancer (UICC, 2002), 4 patients had stage II tumors, 12 patients had stage III tumors and 12 patients had stage IV tumors.

\section{PTV dose}

The averaged volumes of these randomized selected twenty eight patients were 184.4 cc (ranged from $53 \mathrm{cc}$ to 502cc) for PTV1and $621.3 \mathrm{cc}$ (ranged from 192.6cc to $975 \mathrm{cc}$ ) for PTV2, respectively. The target dose coverage were compared in Table 2, the results indicated there is no significant statistical discrepancy between $6 \mathrm{FF}$ and $6 \mathrm{FFF}$ in target dose coverage for plan comparison. The examples of representative dose distribution and DVHs for esophageal carcinoma cases are showed in Fig. 1 and Fig. 2.

Table 2. Averaged over 28 patients DVH parameters, homogeneity, uniformity indices for the PTVs and OAR DVH

\begin{tabular}{|c|c|c|c|c|c|c|}
\hline & Parameters & 6 FF-Plan & 6 FFF-Plan & $p$ & $\mathrm{t}$ & $\Delta(\%)$ \\
\hline \multirow{10}{*}{$\begin{array}{l}\text { PTVs } \\
\text { dose }\end{array}$} & PTV1-D ${ }_{2 \%}(\mathrm{cGy})$ & $6416.3 \pm 93.0$ & $6420.4 \pm 78.7$ & 0.578 & -0.56 & $0.08 \%$ \\
\hline & PTV1-D98\%(cGy) & $5933.0 \pm 102.5$ & $5925.1 \pm 104.5$ & 0.478 & 0.72 & $-0.13 \%$ \\
\hline & PTV1-V $95(\%)$ & $99.7 \pm 0.5$ & $99.6 \pm 0.5$ & 0.619 & -0.50 & $-0.1 \%$ \\
\hline & PTV1-V $100(\%)$ & $95.95 \pm 2.9$ & $95.8 \pm 3.0$ & 0.655 & 0.45 & $-0.15 \%$ \\
\hline & $\mathrm{HI}$ & $1.06 \pm 0.02$ & $1.060 \pm 0.02$ & 0.475 & -0.72 & $0.14 \%$ \\
\hline & CI95\% & $0.453 \pm 0.13$ & $0.451 \pm 0.12$ & 0.566 & 0.58 & $-0.43 \%$ \\
\hline & PTV2-D98\% (cGy) & $4917.5 \pm 133.44$ & $4869.3 \pm 138.1$ & 0.015 & 2.60 & $-0.98 \%$ \\
\hline & PTV2-V $95(\%)$ & $98.94 \pm 0.7$ & $98.7 \pm 0.9$ & 0.088 & 1.77 & $-0.24 \%$ \\
\hline & PTV2-V $100(\%)$ & $96.8 \pm 2.0$ & $96.1 \pm 2.2$ & 0.008 & 2.88 & $-0.7 \%$ \\
\hline & CI95\% & $0.618 \pm 0.05$ & $0.643 \pm 0.05$ & 0.000 & -4.99 & $3.98 \%$ \\
\hline \multirow{10}{*}{$\begin{array}{l}\text { OARs } \\
\text { dose }\end{array}$} & Bilateral lung & & & & & \\
\hline & V30Gy(\%) & $12.4 \pm 4.1$ & $11.7 \pm 3.5$ & 0.008 & 2.85 & $-0.64 \%$ \\
\hline & V20Gy(\%) & $24.8 \pm 7.6$ & $24.3 \pm 6.5$ & 0.240 & 1.20 & $-0.5 \%$ \\
\hline & V10Gy(\%) & $52.3 \pm 14.3$ & $52.4 \pm 14.0$ & 0.883 & -0.15 & $0.16 \%$ \\
\hline & Heart & & & & & \\
\hline & V40Gy(\%) & $12.1 \pm 9.4$ & $10.6 \pm 8.8$ & 0.008 & 2.88 & $-1.48 \%$ \\
\hline & V30Gy(\%) & $25.3 \pm 19.4$ & $23.1 \pm 18.3$ & 0.013 & 2.67 & $-2.16 \%$ \\
\hline & Dmean(cGy) & $1901.8 \pm 1010.9$ & $1840.6 \pm 1015.6$ & 0.008 & 2.85 & $-3.22 \%$ \\
\hline & Spinal cord & & & & & \\
\hline & D1cc(cGy) & $4217.9 \pm 238.8$ & $4210.7 \pm 221.5$ & 0.638 & 0.48 & $-0.17 \%$ \\
\hline \multirow{3}{*}{$\begin{array}{l}\text { Peripheral } \\
\text { doses } \\
\text { volume }\end{array}$} & V45Gy(cc) & $789 \pm 440$ & $738 \pm 414$ & 0.000 & 4.77 & $-6.46 \%$ \\
\hline & V30Gy(cc) & $1984 \pm 804$ & $1822 \pm 718$ & 0.000 & 4.48 & $-8.18 \%$ \\
\hline & V20Gy(cc) & $3510 \pm 1213$ & $3355 \pm 1129$ & 0.000 & 4.28 & $-4.40 \%$ \\
\hline \multirow{4}{*}{$\begin{array}{l}\text { Delivery } \\
\text { efficiency }\end{array}$} & MUs & $594.5 .19 \pm 150.5$ & $724.2 \pm 191.3$ & 0.000 & -7.87 & $21.83 \%$ \\
\hline & Time & $109.1 \pm 12.8$ & $96.1 \pm 9.93$ & 0.000 & & $-11.9 \%$ \\
\hline & & & & & 10.58 & \\
\hline & Mean Dose Rate & $323.3 \pm 47.72$ & $424.5 \pm 82.5$ & 0.000 & -10.4 & $31.3 \%$ \\
\hline
\end{tabular}
parameters with $\mathrm{p}$ - and $\mathrm{t}$ - values for comparison.

\section{OAR dose}

For esophageal carcinoma, the bilateral lung, heart and spinal cord are priority considered 
constrained organs in OARs. The key parameters comparison listed in Table 2, the result showed there is no significant discrepancy between plans with $6 \mathrm{FF}$ beam and 6FFF beam. Only the mean dose of heart is a slightly decreasing with 6 FFF compared to 6FF mode.

\section{Peripheral doses volume}

For peripheral dose around PTV2, a significant reduction of $\mathrm{V}_{45 \mathrm{~Gy}}, \mathrm{~V}_{30 \mathrm{~Gy}}$ and $\mathrm{V}_{20 \mathrm{~Gy}}$ were observed on average by $6.46 \%, 8.18 \%$ and $4.40 \%$, respectively.
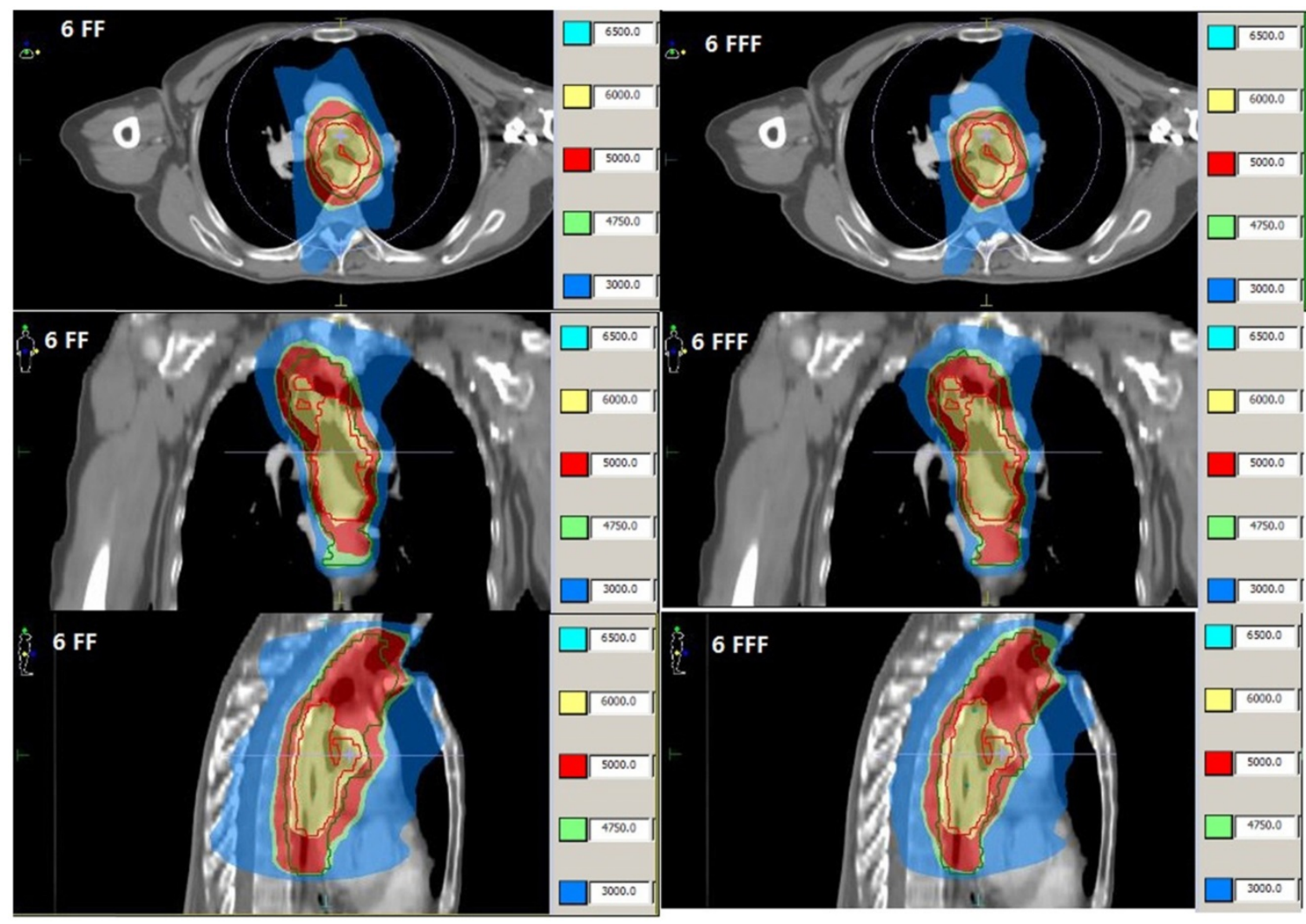

Fig.1. Dose distributions from VMAT of patient 3 with FF beam (Left) and FFF beam (Right).

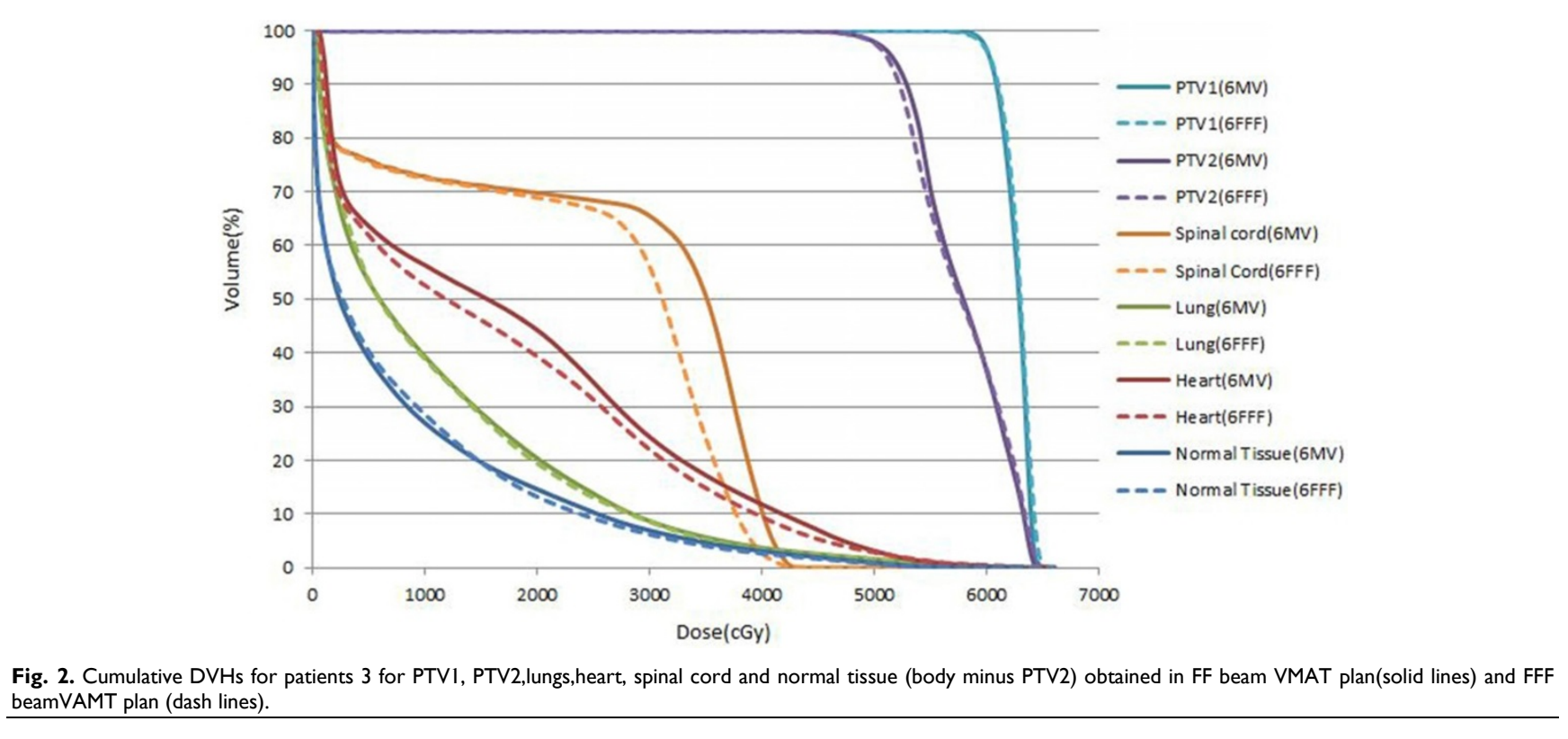

Fig. 2. Cumulative DVHs for patients 3 for PTV1, PTV2,lungs, heart, spinal cord and normal tissue (body minus PTV2) obtained in FF beam VMAT plan(solid lines) and FFF beamVAMT plan (dash lines).

\section{Delivery efficiency comparison}

Generally, the 6 FFF plans have a significantly higher numbers of MUs by $21.83 \%$ compared to $6 \mathrm{FF}$ plans, in contrast, the a significantly reduction of delivery time of $11.90 \%$ was observed for 6 FFF plans.

\section{Discussion}

For comparison convenient, the specific points on the DVH curve, $\mathrm{HI}$ and $\mathrm{CI}$ index were chosen for 
evaluation of treatment plan quality in this study. Table 2 showed that both FF beams and FFF beams can achieve a comparable dose distribution in targets. The $\mathrm{D}_{2 \%}$ which indicated hot dose in targets were 6416.3cGy and 6420.4cGy for FF beams and FFF beams, respectively, both bellowed $108 \%$ of prescripts dose. While the percentage volume of prescripts dose covered were much similar both for PTV1 and PTV2, with FF beams and FFF beams. Only the CI index in PTV2 was significant different, the averaged FFF plans CI index was 3.98\% higher than FF beam plans mainly due to reduction of volume covered by the dose $\left(\mathrm{V}_{95 \%}\right)$ outside PTV2.

For esophagus carcinoma, the pulmonary toxicity is a serious possible side-effect of treatment. So the dose constrain to bilateral lung is priority to consider for evaluation of treatment plans. Numerous literatures had been published with conflicting parameters for prediction of pulmonary toxicity $[13,14]$, but it was still quite difficult to make comparison between studies. In this study, the $\mathrm{V}_{10 G \mathrm{G}}$, $V_{20 G y}$ and $V_{30 G y}$ were used for evaluation of pulmonary toxicity. Mary V.G stated when the total lung $\mathrm{V}_{20 \mathrm{~Gy}}$ is $<25 \%$, the very low risk of pneumonitis would occur [15]. For these two contrast plan groups with FF beams and FFF beams, the average values of $\mathrm{V}_{20 \mathrm{~Gy}}$ and $\mathrm{V}_{30 \mathrm{~Gy}}$ are both very close, meanwhile, the $\mathrm{V}_{20 \mathrm{~Gy}}$ is less than $30 \%$ and $\mathrm{V}_{30 \mathrm{~Gy}}$ is less than $20 \%$, they are all within dose constrain tolerance and no significant statistic difference for both plans. But this isn't indicating that there isn't a significant difference in special case such showed in Fig. 1.

The heart dose constrain is the second considered organ for esophagus carcinoma [16]. Plenty of studies have observed substantial radiation-induced heart disease when the heart received more than $40 \mathrm{~Gy}$ and that the reduction of $\mathrm{V}_{40 \mathrm{~Gy}}$ was pertinent in reducing heart toxicities [17]. In this study, the $\mathrm{V}_{40 \mathrm{~Gy}}$ of FFF beam plan was $10.63 \%$, a slightly decreasing compared to FF beam plan of $12.1 \%$. Wei et al stated that pericardial effusion occurred for patients only $13 \%$ of the time when $V_{30 G y}$ to the heart was kept below $46 \%$ [18]. In this study, the average $\mathrm{V}_{30 \mathrm{~Gy}}$ for the heart were $25.3 \%$ and $23.1 \%$ for FF beams and FFF beams, respectively. They are all below $46 \%$. Although the $\mathrm{V}_{40 G \mathrm{G}}, \mathrm{V}_{30 \mathrm{~Gy}}$ of FFF beams plans had a slightly decreasing trend compared to the FF beams plans, they were all within 3\%. However, the average mean dose of the heart had significant difference, the FFF beam plans was bellowed 3.22\% compared to FF beam plans.

Spinal cord is a dose strict constrained organ for esophagus carcinoma, it also should be spared from target when radiation fields involving the neck, thorax, abdomen and pelvis. John P.K et al stated that a total spinal cord dose of 50 Gy, 60 Gy, and $~ 69$ Gy were associated with a $0.2 \%, 6 \%$ and $50 \%$ rate of myelopathy for conventional fraction dose of 2 Gy per day [19]. In our clinic practice, the dose constrain for spinal cord was that the $D_{\max }$ less than 50 Gy or $D_{1 c c}$ less than $45 \mathrm{~Gy}$. In this study, the average $\mathrm{D}_{1 \mathrm{cc}}$ of spinal cord were 42.18 Gy for FF beam plan and 42.11 Gy for FFF beam plan, respectively. There is no significant difference between these two type beam plans.

Peripheral dose around PTV2 were significant different. For FFF beam plans, the peripheral dose such as $\mathrm{V}_{45 \text { Gy, }} \mathrm{V}_{30 \text { Gy }}$ and $\mathrm{V}_{20 \mathrm{~Gy}}$ were reduced by $6.46 \%$, $8.18 \%$ and $4.40 \%$, respectively compared to the FF beam plans. The organs at risk, such as lung, heart, stomach, trachea, and throat, spinal cord as so on which located around target would be benefit from peripheral dose reduction. Gabriele Kragl et al stated that the peripheral dose were in general smaller for treatment plans calculated with FFF beams [9]. They also measured the dose with $20 \mathrm{~cm}$ distance from the field edge, the result showed that the FFF beam reduced dose about $23 \%$ on average for SBRT plans compared with $6 \mathrm{FF}$ beams. There are many reasons contributed to the peripheral dose reduction. For FFF beam mode, the major source of scattered radiation was removed. It has a great impact on the field outside dose exposure. The scanned profiles showed that FFF beam had the same trend as for FF beam in penumbra region, but the dose fall-off was more pronounced and the values were generally lower for FFF beams. In Vassiliev et al studies, they observed the peripheral dose reduction of $15 \%$ at a depth of 5 $\mathrm{cm}$ and $2 \mathrm{~cm}$ distance from static $4 \times 4 \mathrm{~cm}^{2}$ field edge [20]. The dose fall-off also was different and the values were generally lower for FFF beams. In fact, the small fields are more likely to show a distinct reduction of peripheral dose [21].

As reported in most previously studies, the time reduction and MUs numbers increasing were also found in our plan comparison [22,23]. The MUs numbers increasing were mainly due to achieve a homogeneous dose distribution the FFF beam shape needs to be compensated by a higher number of small segments and MUs. The delivery time reduction mainly resulted from the higher mean dose rates delivery. However, the delivery time were restricted by gantry rotation speed and MLC leaf movement speed. In our studies, the average delivery dose rates were higher $31.32 \%$ compared to $323.3 \mathrm{MU} / \mathrm{min}$ of FF beams plans. Ines Lohse et al [24] irradiated U87-MG and T98G cells using 10 MV FF beams and FFF beams at same dose level using different dose rates, the result indicated that the higher the dose rates, the more decreasing the cell surviving fraction. They also 
irradiated these cells at different dose levels using a dose rates of $400 \mathrm{MU} / \mathrm{min}$, the results showed that they couldn't see any difference in cell kill at a dose level of $5 \mathrm{~Gy}$, but a statistically significant reduction of clonogenic survival were observed compared to the treatment with FF beams at a dose of $10 \mathrm{~Gy}$, and the higher the single dose, the more pronounced the cancer cell survival diverges within two beams. The result indicated that the use of the FFF beam more efficiently decreases tumor cell survival. This phenomenon can benefit for higher dose SBRT regiments.

However, the plan comparison was conducted by same optimization parameters settings. This could limit the plans quality. In future work, the effort to reduce the numbers of MUs and segments of FFF beams could be developed, this can go step further to decrease the peripheral dose around target.

\section{Conclusion}

For esophagus carcinoma, the most distinctive character of FFF beam plans was reduction of peripheral dose around targets while the dose distribution in targets were comparable, this can benefit for the OARs located around targets.

\section{Competing Interests}

The authors have declared that no competing interest exists.

\section{References}

1. Mariette C, Piessen G, Triboulet JP. Therapeutic strategies in oesophageal carcinoma: role of surgery and other modalities. Lancet Oncol. 2007; 8(6):545-53.

2. Van Meerten E, van der Gaast A. Systemic treatment for oesophageal cancer. Eur J Cancer. 2005; 41(5): 664-72.

3. Nicholas H, Wolfgang AT, Kerwyn FF, et al. Comparison of Prostate IMRT and VMAT Biologically Optimised Treatment Plans. Medical Dosimetry. 2011; 36(3):292-298

4. Liu HY, Chen XD, He ZJ, et al. Evaluation of 3D-CRT, IMRT and VMAT radiotherapy plans for left breast cancer based on clinical dosimetric study. Computerized Medical Imaging and Graphics. 2016; 54:1-5.

5. Xu,LM ,Kang ML, Jiang B, et al. A study of the dosimetric characteristics between different fixed-field IMRT and VMAT in early-stage primary mediastinal B-cell lymphoma. Medical Dosimetry. 2018; 43(1):91-99.

6. Teoh M, Clark CH, Wood K, et al. Volumetric modulated arc therapy: a review of current literature and clinical use in practice. The British Journal of Radiology, 2011; 84(1007):967-996.

7. Pichandia A, Kadirampatti MG, Jerin A, et al. Analysis of physical parameters and determination of inflection point for Flattening Filter Free beams in medical linear accelerator. reports of practical oncology and radiotherapy. 2014; 19(5):322-331.

8. Stock M, Kontrisova K, Dieckmann K, et al. Development and application of a real-time monitoring and feedback system for deep inspiration breath-hold based on external marker tracking. Med Phys. 2006; 33(8):2868-77.

9. Krag G, Baier F, Lutz S, et al. Flattening filter free beams in SBRT and IMRT: Dosimetric assessment of peripheral doses. Z Med. Phys. 2011; 21(2):91-101.

10. Laure V, Sandra B, Monia O, et al. Dosimetric comparison of flattened and unflattened beams for stereotactic body radiation therapy: Impact of the size of the PTV on dynamic conformal arc and volumetric modulated arc therapy. Physica Medica. 2016; 32(11):1405-1414.

11. Liam VB, Lee H, Matthew BP. Volumetric Modulated Arc Therapy vs IMRT for the Treatment of Distal Esophageal Cancer. Medical Dosimetry. 2011; 36(4):404-409.

12. Chen $\mathrm{H}$, Wang $\mathrm{H}, \mathrm{Gu} \mathrm{HL}$, et al. Study for reducing lung dose of upper thoracic esophageal cancer radiotherapy by auto-planning: volumetric-modulated arc therapy vs intensity-modulated radiation therapy. Medical Dosimetry. 2018; 43(3):243-250.
13. Kim TH, Cho KH, Pyo HR, et al. Dose-volumetric parameters for predicting severe radiation pneumonitis after three-dimensional conformal radiation therapy for lung cancer. Radiology. 2005; 235(1):208-215.

14. Kong FM, Hayman JA, Griffith KA, et al. Final toxicity results of a radiation-dose escalation study in patients with non-small-cell lung cancer (NSCLC): predictors for radiation pneumonitis and fibrosis. Int J Radiat Oncol Biol Phys. 2006; 65(4):1075-1086.

15. Mary VG, James AP, Bahman E, et al. Clinical Dose-Volume Histogram analysis for pneumonitis after 3D treatment for non-small cell cancer (NSCLC). Int J Radiation Oncol Biol Phys. 1999; 45(2):323-329.

16. Jannet CB, Peter L, Joachim $\mathrm{W}$, et al. Is cardiac toxicity a relevant issue in the radiation treatment of esophageal cancer? Radiotherapy and Oncology. 2015; 114(1): 85-90.

17. Ishikura $\mathrm{S}$, Nihei $\mathrm{K}$, Ohtsu $\mathrm{A}$, et al. Long-term toxicity after definitive chemo-radiotherapy for squamous cell carcinoma of the thoracic esophagus. J Clin Oncol. 2003; 21(14):2697-2702.

18. Wei X, Liu HH, Tucker SL, et al. Risk factors for pericardial effusion in inoperable esophageal cancer patients treated with definitive chemoradiation therapy. Int J Radiat Oncol Biol Phys. 2008; 70(3):707-714.

19. John PK, Alabert J.Van DK, Timothy ES, et al. Radiation Dose-Volume effects in the spinal cord. Int J Radiation Oncology Biol Phys. 2010; 76(Suppl 3):S42-S49.

20. Vassiliev ON, Titt U, Pönisch F, Kry SF, et al. Dosimetric properties of photon beams from a flattening filter free clinical accelerator. Phys Med Biol. 2006; 51(7):1907-1917.

21. Titt U, Vassiliev ON, Pönisch F, et al. A flattening filter free photon treatment concept evaluation with Monte Carlo. Med Phys. 2006; 33(6):1595-1602.

22. Wolfgang L, Gabriele K, Dietmar G. Evaluation of treatment plan quality of IMRT and VMAT with and without flattening filter using Pareto optimal fronts. Radiotherapy and Oncology. 2013; 109(3):437-441.

23. Zhuang MZ, Zhang TD, Chen ZJ, et al. Volumetric modulation arc radiotherapy with flattening filter-free beams compared with conventional beams for nasopharyngeal carcinoma: a feasibility study. Chinese Journal of Cancer. 2013; 32(7):397-402.

24. Ines L, Stephanie L, Jan H, et al. Effect of high dose per pulse flattening filter-free beams on cancer cell survival. Radiotherapy and Oncology. 2011; 101(1):226-232. 